\title{
Evaluation of the Asymmetric Voigt Profile and Complex Error Functions in Terms of the Kummer Functions
}

\author{
H.O. Di Rocco And M. Aguirre TÉllez \\ Instituto de Física Arroyo Seco and Núcleo de Matemática Pura y Aplicada \\ Facultad de Ciencias Exactas, Universidad Nacional del Centro \\ Pinto 399, 7000 Tandil, Argentina
}

(Received August 16, 2004; in final form October 19, 2004)

\begin{abstract}
In this work we present the explicit representations of the Voigt function $K(a, b)$ (the convolution between a Gaussian and a Lorentzian function), the function $N(a, b)$ defined as the convolution of Gaussian and dispersion distributions as well as the complex error function $\operatorname{erf}(a+\mathrm{i} b)$, all in terms of the Kummer functions $M\left(\alpha, \gamma, a^{2}\right)$. The expansions are valid for all values of the parameter $a$ (the relation between Lorentzian and Gaussian widths at the half maxima). Previous analytical works were known only when the parameter $a \leq 1$, or were based on numerical interpolations or empirical approximations. Also, new series and asymptotic expansions are presented.
\end{abstract}

PACS numbers: 32.70.Jz, 33.70.Jg, 34.20.Cf

\section{Introduction}

The Voigt function $K(a, b)$ and the complex error function $\operatorname{erf}(a+\mathrm{i} b)$ are important in diverse branches of physics and applied mathematics; $K(a, b)$ is the convolution between Gaussian and Lorentzian probability distributions. It is useful in physical sciences whenever the two distributions arise independently. In fact, it is used in the study of terrestrial and stellar atmospheres, in atomic and plasma physics (analysis of symmetric and asymmetric line profiles [1]) as well as in the physics of nuclear-fission reactors. Jointly with the function $N(a, b)$, they are important in the theory of matter-radiation interaction [2]. From the practical point of view, many techniques were devised for computing $K(a, b)$ more 
or less efficiently. $K(a, b)$ is related to the complex error function $\operatorname{erf}(a+\mathrm{i} b)$; see below, Eq. (8) and this relation is the basis of several algorithms to compute it [3]. Researchers did not find a closed-form expression for $K(a, b)$ for all values of the parameter $a$ (see below, Eq. (4)) and the expression has been treated from the graphical and numerical points of view. Many empirical approximations and computational procedures were (and are) currently published in diverse physical journals. Moreover, one of them is devoted exclusively to the problems of radiative transfer, where the Voigt function is of paramount interest [4].

In this work, we present explicit representations of $K(a, b), N(a, b)$, and $\operatorname{erf}(a+\mathrm{i} b)$ in terms of the Kummer functions $M\left(\alpha, \gamma, a^{2}\right)$, for all values of the parameter $a$. This is interesting because some computer algebra systems (CAS, eg. Maple) contain the programmed Kummer functions. The more relevant results are presented in Eqs. $(14,16,26)$ and (28). Furthermore, we deduce other integral representations for $W(z)=\exp \left(z^{2}\right)(1-\operatorname{erf}(z)) / \sqrt{\pi}$ (see Eqs. (8) and (18)). Finally, several new series and asymptotic expansions, useful for numerical calculations, are presented in diverse Appendixes.

\section{The Voigt function, first notation and different expressions}

There is a wide and confusing variety of notations for the probability distributions; we use the normalized expressions for $G(x), L(x)$, and $V(x)$ which make the area under the curves equal to the unity. Despite other definitions [5], we write explicitly the parameter related to the half width at half maximum (HWHM). The following notation is employed: for the moment, $x$ is the independent variable (see below, one row before Eq. (6); furthermore, $w$ is used for the parameter giving the simplest normalized expressions and $\gamma$ for the HWHM's; lower index G, L, and V, indicate Gauss, Lorentz, and Voigt, respectively.

With the above conventions, the normalized Gaussian distribution is

$$
G(x)=G_{0} \exp \left[-\left(x / w_{\mathrm{G}}\right)^{2}\right],
$$

where $w_{\mathrm{G}}=\gamma_{\mathrm{G}} / \sqrt{\ln 2}$ and $G_{0}=1 / \sqrt{\pi} w_{\mathrm{G}}$. Analogously, the Lorentzian (or Cauchy) distribution is given by

$$
L(x)=L_{0} \frac{w_{\mathrm{L}}^{2}}{\left(x^{2}+w_{\mathrm{L}}^{2}\right)},
$$

where $w_{\mathrm{L}}=\gamma_{\mathrm{L}}$ and $L_{0}=1 / \pi w_{\mathrm{L}}$. From Eqs. (1) and (2), the convolution between both functions is given by the normalized expression

$$
V(x)=\frac{\left(w_{\mathrm{L}} / w_{\mathrm{G}}\right)}{\pi^{3 / 2}} \int_{-\infty}^{\infty} \frac{\mathrm{e}^{-\left(x^{\prime} / w_{\mathrm{G}}\right)^{2}}}{\left(x-x^{\prime}\right)^{2}+w_{\mathrm{L}}^{2}} \mathrm{~d} x^{\prime} .
$$

Defining the dimensionless parameter $a$ as

$$
a=w_{\mathrm{L}} / w_{\mathrm{G}} \equiv \sqrt{\ln 2} \gamma_{\mathrm{L}} / \gamma_{\mathrm{G}}
$$

and the dimensionless variables $y, b$, and $u$ as 


$$
y=x^{\prime} / w_{\mathrm{G}}, \quad b=x / w_{\mathrm{G}}, \quad u=(y-b) / a,
$$

we can write the normalized function $K_{a}(b) \equiv w_{\mathrm{G}} V_{a}(b)$ in the forms

$$
K_{a}(b)=\frac{a}{\pi^{3 / 2}} \int_{-\infty}^{\infty} \frac{\mathrm{e}^{-y^{2}} \mathrm{~d} y}{(y-b)^{2}+a^{2}}=\frac{1}{\pi^{3 / 2}} \int_{-\infty}^{\infty} \frac{\mathrm{e}^{-(a u+b)^{2}} \mathrm{~d} u}{u^{2}+1} .
$$

After some manipulations, it can be shown that Eq. (6) is equivalent to

$$
K_{a}(b)=\frac{1}{\pi a} \int_{0}^{\infty} \mathrm{e}^{-x} \mathrm{e}^{-(x / 2 a)^{2}} \cos (b x / a) \mathrm{d} x .
$$

Equation (7) is the basis of all our results.

\section{The change of notation: the Voigt and dispersion functions, $K(a, b)$ and $N(a, b)$, respectively}

As indicated in Ref. [3], Eq. (6) can be manipulated in terms of the complementary error function of complex argument as:

$$
K_{a}(b)=\operatorname{Re} W(z) \equiv \operatorname{Re}\left[\mathrm{e}^{z^{2}}(1-\operatorname{erf}(z)) / \sqrt{\pi}\right],
$$

where $[6]$

$$
z=a+\mathrm{i} b \quad \text { and } \quad \operatorname{erf}(a+\mathrm{i} b)=\frac{2}{\sqrt{\pi}} \int_{0}^{a+\mathrm{i} b} \exp \left(-t^{2}\right) \mathrm{d} t,
$$

$t$ being also a complex variable. Therefore, after Eqs. (8) and (9), the parameter " $a$ " can be considered as a variable and we can change the notation

$$
K(a, b) \equiv K_{a}(b)
$$

The relation given in Eq. (8) is one of the methods used to devise algorithms to compute $K(a, b)$ from series and asymptotic expansions of $\operatorname{erf}(z)[3]$. In this work we proceed in the reciprocal form. Writing $W(z) \equiv \mathrm{e}^{z^{2}}(1-\operatorname{erf}(z)) / \sqrt{\pi}$ in the form

$$
W(z)=K(a, b)+\mathrm{i} N(a, b),
$$

we found representations of $K(a, b)$ and $N(a, b)$ in terms of the Kummer (or confluent hypergeometric) functions, $M\left(\alpha, \gamma, a^{2}\right)[7]$ :

$$
M\left(\alpha, \gamma, a^{2}\right)=1+\frac{\alpha}{\gamma} a^{2}+\frac{\alpha(\alpha+1)}{\gamma(\gamma+1)} \frac{a^{4}}{2 !}+\frac{\alpha(\alpha+1)(\alpha+2)}{\gamma(\gamma+1)(\gamma+2)} \frac{a^{6}}{3 !}+\ldots,
$$

and then we evaluate $\operatorname{Re}(\operatorname{erf}(z))$ and $\operatorname{Im}(\operatorname{erf}(z))$ in terms of $M\left(\alpha, \gamma, a^{2}\right)$.

It is important to remark that, despite the variety of definitions, we have verified that Eqs. $(3,6,7)$ and $(8)$ are consistent between them. 


\section{Expansion of $K(a, b)$ and $N(a, b)$ for all values of $a$} in terms of $M\left(\alpha, \gamma, a^{2}\right)$.

The first step toward the attainment of a compact expression for the Voigt function is the expansion of $K(a, b)$ in a power series valid for all values of the parameter $a$. From Eq. (7) and using the following representation:

$$
\cos (b x / a)=\sum_{n=0}^{\infty} \frac{(-1)^{n}}{(2 n) !}\left(\frac{b}{a}\right)^{2 n} x^{2 n},
$$

we can write

$$
K(a, b)=\frac{1}{\pi a} \sum_{n=0}^{\infty} \frac{(-1)^{n}}{(2 n) !}\left(\frac{b}{a}\right)^{2 n} I_{n}(a),
$$

with

$$
I_{n}(a)=\int_{0}^{\infty} \mathrm{e}^{-x} \mathrm{e}^{-(x / 2 a)^{2}} x^{2 n} \mathrm{~d} x .
$$

The above integral can be expressed in terms of $M\left(\alpha, \gamma, a^{2}\right)[8]$ :

$$
\begin{aligned}
I_{n}(a) & =2^{2 n} a\left[a^{2 n} \Gamma\left(\frac{2 n+1}{2}\right) M\left(\frac{2 n+1}{2}, \frac{1}{2}, a^{2}\right)\right. \\
& \left.-2 a^{2 n+1} \Gamma(n+1) M\left(n+1, \frac{3}{2}, a^{2}\right)\right] /\left[\Gamma(n+1) \Gamma\left(\frac{2 n+1}{z}\right)\right]
\end{aligned}
$$

such that

$$
K(a, b)=\frac{1}{\sqrt{\pi}} \sum_{n=0}^{\infty}(-1)^{n} k_{n}(a) b^{2 n} ;
$$

explicitly

$$
\begin{aligned}
& K(a, b)=\frac{1}{\sqrt{\pi}} \sum_{n=0}^{\infty}(-1)^{n} \\
& \times\left[\frac{\Gamma\left(\frac{2 n+1}{2}\right) M\left(\frac{2 n+1}{2}, \frac{1}{2}, a^{2}\right)-2 a \Gamma(n+1) M\left(n+1, \frac{3}{2}, a^{2}\right)}{\Gamma(n+1) \Gamma\left(\frac{2 n+1}{2}\right)}\right] b^{2 n} .
\end{aligned}
$$

On the other hand, taking into account that $W(z)$ satisfy the CauchyRiemann conditions, we obtain $N(a, b)$ from

$$
N(a, b)=\int\left(\frac{\partial K(a, b)}{\partial a}\right) \mathrm{d} b .
$$

Deriving Eq. (14), remembering that

$$
\frac{\mathrm{d} M\left(\alpha, \gamma, x^{2}\right)}{\mathrm{d} x}=\frac{2 \alpha x M\left(\alpha+1, \gamma+1, x^{2}\right)}{\gamma}
$$

integrating and taking into account the well-known properties of the Kummer 
functions [8], we obtain

$$
\begin{gathered}
N(a, b)=-\frac{b}{\sqrt{\pi}} \sum_{n=0}^{\infty}(-1)^{n}\left[\frac{1}{\Gamma(n+3 / 2)} M\left(n+1, \frac{1}{2}, a^{2}\right)\right. \\
\left.-\frac{2 a}{\Gamma(n+1)} M\left(n+\frac{3}{2}, \frac{3}{2}, a^{2}\right)\right] b^{2 n} .
\end{gathered}
$$

The same result can be obtained by developing $-\sin (b x / a)$ in Eq. (7) in place of $\cos (b x / a)$. Then, remembering Eq. (10), we can say that

$$
\begin{gathered}
W(z)=\frac{1}{\pi a} \int_{0}^{\infty} \mathrm{e}^{-x} \mathrm{e}^{-(x / 2 a)^{2}}[\cos (b x / a)-\mathrm{i} \sin (b x / a)] \mathrm{d} x \\
=\frac{1}{\pi a} \int_{0}^{\infty} \mathrm{e}^{-x} \mathrm{e}^{-(x / 2 a)^{2}} \mathrm{e}^{-\mathrm{i} b x / a} \mathrm{~d} x,
\end{gathered}
$$

which is an integral representation of $W(z)$. Furthermore, making the variable change $x=a t$, and as $z=a+\mathrm{i} b$, other integral representation of $W(z)$ is

$$
W(z)=\frac{1}{\pi} \int_{0}^{\infty} \mathrm{e}^{-z t} \mathrm{e}^{-t^{2} / 4} \mathrm{~d} t
$$

considered for some authors [1]. However, to our knowledge, Eq. (17) was not previously reported.

\section{Real and imaginary parts of the complex error function}

Starting of Eq. (10) and writing

$$
1-\operatorname{erf}(z)=C(a, b)+\mathrm{i} D(a, b) \quad \text { so } \quad \operatorname{erf}(z)=[1-C(a, b)]-\mathrm{i} D(a, b)
$$

we obtain

$$
\sqrt{\pi} \mathrm{e}^{\left(b^{2}-a^{2}\right)} K(a, b)=[C(a, b) \cos (2 a b)-D(a, b) \sin (2 a b)] \equiv W_{1}(a, b)
$$

and

$$
\sqrt{\pi} \mathrm{e}^{\left(b^{2}-a^{2}\right)} N(a, b)=[D(a, b) \cos (2 a b)+C(a, b) \sin (2 a b)] \equiv W_{2}(a, b) ;
$$

then

$$
C(a, b)=W_{1}(a, b) \cos (2 a b)+W_{2}(a, b) \sin (2 a b)
$$

and

$$
D(a, b)=W_{2}(a, b) \cos (2 a b)-W_{1}(a, b) \sin (2 a b) .
$$

Therefore, the real and imaginary parts of $\operatorname{erf}(z)$ are

$$
\begin{aligned}
& \operatorname{Re}(\operatorname{erf}(z))=1-C(a, b) \\
& \quad=1-\sqrt{\pi} \mathrm{e}^{\left(b^{2}-a^{2}\right)}[K(a, b) \cos (2 a b)+N(a, b) \sin (2 a b)]
\end{aligned}
$$


and

$$
\begin{aligned}
& \operatorname{Im}(\operatorname{erf}(z))=-D(a, b) \\
& \quad=-\sqrt{\pi} \mathrm{e}^{\left(b^{2}-a^{2}\right)}[N(a, b) \cos (2 a b)-K(a, b) \sin (2 a b)]
\end{aligned}
$$

with $K(a, b)$ and $N(a, b)$ given by Eq. (14) and Eq. (16), respectively.

\section{Appendix 1: Expansions of $K(a, b)$ and $N(a, b)$ for $a<1$ and $a>1$}

Although the expressions given by Eqs. (14) and (16) are valid $\forall a$, for completeness we present the following expansions for $a<1$ and $a>1$. Equations (27-29) are new, to our knowledge.

$$
\text { 6.1. } K(a, b) \text {; expansions for } a<1 \text { and } a>1
$$

The fundamental relation for all evaluations is Eq. (7). In fact, when $a<1$, after replacing

$$
\mathrm{e}^{-x}=\sum_{n=0}^{\infty}(-1)^{n} \frac{x^{n}}{n !}
$$

in that equation, we obtain the rapidly convergent expression (see Eq. 3.952-8 of Ref. [8]):

$$
K(a<1, b)=\frac{\mathrm{e}^{-b^{2}}}{\pi} \sum_{n=0}^{\infty} \frac{(-1)^{n}(2 a)^{n}}{n !} \Gamma\left(\frac{n+1}{2}\right) M\left(\frac{-n}{2}, \frac{1}{2}, b^{2}\right) .
$$

This is a better known case because is widely used in astrophysics [9].

For $a>1$ there are no published analytical results valid for all values of the variable $b$, although it is of interest in plasma physics. After the expansion

$$
\mathrm{e}^{-(x / 2 a)^{2}}=\sum_{n=0}^{\infty}(-1)^{n} \frac{x^{2 n}}{n !(2 a)^{2 n}}
$$

and using the results of the cosine transform (Eq. 3.944-6 of Ref. [8]), we obtain the asymptotic expansion

$$
K(a>1, b) \sim \frac{1}{\pi \sqrt{b^{2}+a^{2}}} \sum_{n=0}^{\infty} \frac{(-1)^{n}(2 n) !}{2^{2 n} n !\left(b^{2}+a^{2}\right)^{n}} \cos [(2 n+1) \arctan (b / a)],
$$

that we will use below.

$$
\text { 6.2. } N(a, b) \text {; expansions for } a<1 \text { and } a>1
$$

As was indicated after Eq. (16), $N(a, b)$ can be represented by an expression similar to Eq. (7), by replacing $\cos (b x / a)$ by $-\sin (b x / a)$ :

$$
N(a, b)=\frac{-1}{\pi a} \int_{0}^{\infty} \mathrm{e}^{-x} \mathrm{e}^{-(x / 2 a)^{2}} \sin (b x / a) \mathrm{d} x
$$


Then, working as in the above paragraph and using the results for the sine transform, we obtain for $a \leq 1$

$$
\begin{aligned}
N(a & \leq 1, b \rightarrow \infty)=-\frac{2 b \mathrm{e}^{-b^{2}}}{\pi} \\
& \times \sum_{n=0}^{\infty} \frac{(-1)^{n}(2 a)^{n}}{n !} \Gamma\left(\frac{n+2}{2}\right) M\left(\frac{1-n}{2}, \frac{3}{2}, b^{2}\right),
\end{aligned}
$$

whereas for $a>1$

$$
\begin{aligned}
N(a & >1, b)=-\frac{1}{\pi \sqrt{b^{2}+a^{2}}} \\
& \times \sum_{n=0}^{\infty} \frac{(-1)^{n}(2 n) !}{2^{2 n} n !\left(b^{2}+a^{2}\right)^{n}} \sin [(2 n+1) \arctan (b / a)] .
\end{aligned}
$$

7. Appendix 2: expressions of $K(a, b)$ and $N(a, b)$ for $a=0$ and $a \rightarrow \infty$

$$
\text { 7.1. } K(a, b)
$$

We note some limits for the integral expression in Eq. (3). As $a \rightarrow 0$, the Voigt integral tends to the Gaussian profile. On the other hand, as $a \rightarrow \infty$, the integral approaches a Lorentzian function. We will see that our expansion (14) satisfies these requirements. In the following, we call $G(b)$ and $L(a, b)$, respectively, to the functions $G(x)$ and $L(x)$, when $w_{\mathrm{G}}=1$ and therefore $w_{\mathrm{L}}=a$.

For $a=0, M\left(\alpha, \gamma, a^{2}\right)=1$ and we get

$$
K(0, b)=\frac{1}{\sqrt{\pi}} \sum_{n=0}^{\infty} \frac{(-1)^{n}}{n !} b^{2 n}=\frac{1}{\sqrt{\pi}} \exp \left(-b^{2}\right),
$$

that is $G(b)$.

For $a \rightarrow \infty$, using the asymptotic expansion for $M(\alpha, \gamma, z)$ (see the last Appendix), $k_{n}$ is

$$
k_{n} \sim \frac{(-1)^{-(n+1)} \sqrt{\pi}}{\Gamma\left(\frac{2 n+1}{2}\right) \Gamma\left(\frac{1-2 n}{2}\right) a^{2 n+1}}
$$

and taking into account that

$$
\frac{1}{\Gamma\left(\frac{2 n+1}{2}\right) \Gamma\left(\frac{1-2 n}{2}\right)}=\frac{(-1)^{n}}{\pi},
$$

we have

$$
K(a \rightarrow \infty, b) \sim \frac{1}{\pi a} \sum_{n=0}^{\infty}(-1)^{n}(b / a)^{2 n}=\frac{1}{\pi} \frac{a}{\left(b^{2}+a^{2}\right)},
$$

that is $L(a, b)$. 


$$
\text { 7.2. } N(a, b)
$$

Analogously to the above section, when $a=0$ and the $M^{\prime} s=1$

$$
N(0, b)=-\frac{1}{\sqrt{\pi}} \sum_{n=0}^{\infty}(-1)^{n} \frac{1}{\Gamma(n+3 / 2)} b^{2 n+1} ;
$$

this sum can be evaluated analytically to

$$
N(0, b)=\frac{\mathrm{i}}{\sqrt{\pi}} \mathrm{e}^{-b^{2}} \operatorname{erf}(\mathrm{i} b)=\frac{-2 D(b)}{\pi},
$$

$D(b)$ being the Dawson function [10]

$$
D(b)=\mathrm{e}^{-b^{2}} \int_{0}^{b} \mathrm{e}^{t^{2}} \mathrm{~d} t .
$$

For $a \rightarrow \infty$, the asymptotic expansions of $M^{\prime} s$ give us

$$
N(a \rightarrow \infty, b)=-\frac{1}{\pi a} \sum_{n=0}^{\infty}(-1)^{n}\left(\frac{b}{a}\right)^{2 n+1},
$$

that evaluates to

$$
N(a \rightarrow \infty, b)=-\frac{b}{\pi\left(a^{2}+b^{2}\right)} .
$$

It is easy to verify that the function given by Eqs. (35) satisfies:

$$
N(a \rightarrow \infty, b)=\int\left(\frac{\partial L(a, b)}{\partial a}\right) \mathrm{d} b .
$$

\section{Appendix 3: asymptotic expansions for $b \rightarrow \infty$}

Although the expressions given by Eqs. (14) and (16) are valid $\forall b$, by numerical reasons it is convenient to use the following expressions for large values of $b$ :

\subsection{Real part}

For $a<1$, we can use Eq. (26), due to the strongly convergent factor $\mathrm{e}^{-b^{2}}$. For $a>1$, it is sufficient to take $n=0,1$ in Eq. (27):

$$
\begin{aligned}
K(a & >1, b \rightarrow \infty) \sim \frac{a}{\pi\left(b^{2}+a^{2}\right)}\left[1-\frac{\cos (3 \arctan (b / a))}{2\left(b^{2}+a^{2}\right) \cos (\arctan (b / a))}\right] \\
& =L(a, b)\left(1-f_{1}(b / a)\right) .
\end{aligned}
$$

\subsection{Imaginary part}

Analogously to the above paragraph, for $a<1$ we can use Eq. (28), whereas for $a>1$, we take $n=0,1$ in Eq. (29): 


$$
\begin{aligned}
N(a & >1, b \rightarrow \infty) \sim-\frac{b}{\pi\left(b^{2}+a^{2}\right)}\left[1-\frac{1}{2} \frac{\sin (3 \arctan (b / a))}{\left(b^{2}+a^{2}\right) \sin (\arctan (b / a))}\right] \\
& =N(a \rightarrow \infty, b)\left(1-f_{2}(b / a)\right) .
\end{aligned}
$$

\section{Appendix 4: evaluation of the coefficients for high values of $a$}

The coefficients of $b^{n}$ in Eqs. (14) and (16) are proportional to $M\left(\alpha, \gamma, a^{2}\right)$. From the numerical point of view, for the evaluation of the Kummer functions of high values of $a$ it is necessary to use the following asymptotic expansion [7]:

$$
\begin{gathered}
M(\alpha, \gamma, z) \sim \frac{\Gamma(\gamma)}{\Gamma(\gamma-\alpha)}(-z)^{-\alpha} G(\alpha, \alpha-\gamma+1,-z) \\
+\frac{\Gamma(\gamma)}{\Gamma(\alpha)} \mathrm{e}^{z}(z)^{\alpha-\gamma} G(\gamma-\alpha, 1-\alpha, z)
\end{gathered}
$$

with

$$
G(a, b, z)=1+\frac{a b}{z}+\frac{a(a+1) b(b+1)}{2 z^{2}}+\ldots
$$

\section{Acknowledgments}

Partial supports from the Universidad Nacional del Centro, the Consejo Nacional de Investigaciones Científicas y Técnicas (CONICET, Argentina) and the Comisión de Investigaciones Científicas (CIC, Provincia de Buenos Aires) are gratefully acknowledged.

Also, the authors acknowledge to Dr. Roman Ciurylo, from Nicolaus Copernicus University (Torun, Poland), for the supply of several published papers.

\section{References}

[1] R. Ciuryło, J. Domysławska, R.S. Trawiński, Acta Phys. Pol. A 83, 425 (1993).

[2] A.E. Siegman, Lasers, 2nd. ed., University Science, Mil Valley, California 1986.

[3] W.J. Thompson, Comput. in Phys. 7, 627 (1993).

[4] R.J. Wells, J. Quant. Spectrosc. Radiat. Transf. 62, 29 (1999).

[5] M. Abramowitz, I.A. Stegun, Handbook of Mathematical Functions, Dover Publications, New York 1968.

[6] A. Erdelyi, W. Magnus, F. Oberhettinger, F.G. Tricomi, Higher Transcendental Functions, Vol. 2, McGraw-Hill, New York 1953.

[7] S.M. Smirnov, A Course of Higher Mathematics, Pergamon Press, Oxford 1964 (translated from Russian). 
[8] I.S. Gradstein, I.M. Ryzhik, Table of Integrals, Series and Products, 5th ed., Academic Press, San Diego 1994.

[9] D. Mihalas, Stellar Atmospheres, 2nd. ed., W.H. Freeman, San Francisco 1982.

[10] W.H. Press, S.A. Teukolsky, W.T. Vetterling, B.P. Flannery, Numerical Recipes, 2nd. ed. Cambridge University Press, Cambridge 1992. 